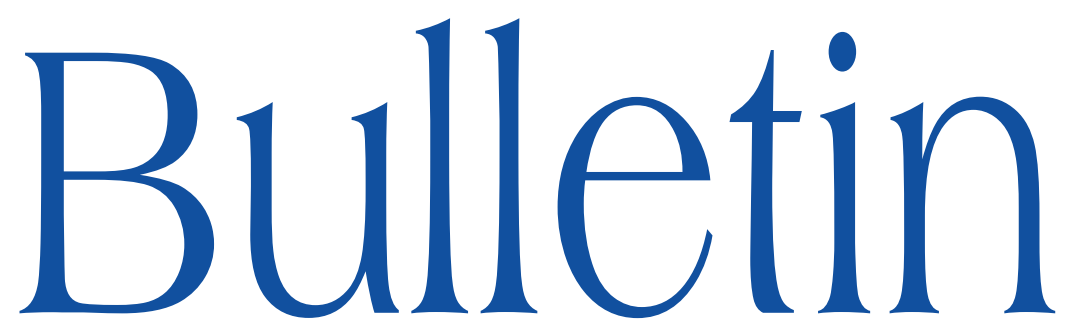

de la SOCIÉTÉ MATHÉMATIQUE DE FRANCE

\title{
COMPATIBILITY OF THE THETA CORRESPONDENCE WITH THE WHITTAKER FUNCTORS
}

Vincent Lafforgue \& Sergey Lysenko

\section{Tome 139} Fascicule 1 


\title{
COMPATIBILITY OF THE THETA CORRESPONDENCE WITH THE WHITTAKER FUNCTORS
}

\author{
By Vincent Lafforgue \& Sergey Lysenko
}

\begin{abstract}
We prove that the global geometric theta-lifting functor for the dual pair $(H, G)$ is compatible with the Whittaker functors, where $(H, G)$ is one of the pairs $\left(\mathrm{SO}_{2 n}, \mathbb{S p}_{2 n}\right),\left(\mathbb{S p}_{2 n}, \mathrm{SO}_{2 n+2}\right)$ or $\left(\mathbb{G L}_{n}, \mathbb{G L}_{n+1}\right)$. That is, the composition of the theta-lifting functor from $H$ to $G$ with the Whittaker functor for $G$ is isomorphic to the Whittaker functor for $H$.

RÉSumé (Compatibilité de la thêta-correspondence avec les foncteurs de Whittaker)

Nous démontrons que le foncteur géométrique de théta-lifting pour la paire duale $(H, G)$ est compatible avec la normalisation de Whittaker, où $(H, G)$ est l'une des paires $\left(\mathrm{SO}_{2 n}, \mathbb{S p}_{2 n}\right),\left(\mathbb{S p}_{2 n}, \mathrm{SO}_{2 n+2}\right)$ ou $\left(\mathbb{G L}_{n}, \mathbb{G L}_{n+1}\right)$. Plus précisément, le composé du foncteur de théta-lifting de $H$ vers $G$ et du foncteur de Whittaker pour $G$ est isomorphe au foncteur de Whittaker pour $H$.
\end{abstract}

We prove in this note that the global geometric theta lifting for the pair $(H, G)$ is compatible with the Whittaker normalization, where $(H, G)=$ $\left(\mathrm{SO}_{2 n}, \mathbb{S p}_{2 n}\right),\left(\mathbb{S p}_{2 n}, \mathrm{SO}_{2 n+2}\right)$, or $\left(\mathbb{G L}_{n}, \mathbb{G L}_{n+1}\right)$. More precisely, let $k$ be an algebraically closed field of characteristic $p>2$. Let $X$ be a smooth projective connected curve over $k$. For a stack $S$ write $\mathrm{D}(S)$ for the derived category of étale constructible $\overline{\mathbb{Q}}_{\ell}$-sheaves on $S$. For a reductive group $G$ over $k$ write

Texte reçu le 3 février 2009, accepté le 26 novembre 2009

Vincent Lafforgue, Laboratoire de Mathématiques - Analyse, Probabilités, Modélisation Orléans (MAPMO), UMR CNRS 6628, Université d'Orléans, Rue de Chartres, B.P. 6759, 45067 Orléans Cedex 2, France

Sergey Lysenko, Institut Élie Cartan Nancy, Université Henri Poincaré Nancy 1, B.P. 239, F-54506 Vandœuvre-lès-Nancy Cedex, France 2000 Mathematics Subject Classification. - 11R39; 14H60.

Key words and phrases. - Geometric Langlands, Whittaker functor, theta-lifting. 
$\operatorname{Bun}_{G}$ for the stack of $G$-torsors on $X$. The usual Whittaker distribution admits a natural geometrization Whit $_{G}: \mathrm{D}\left(\operatorname{Bun}_{G}\right) \rightarrow \mathrm{D}(\operatorname{Spec} k)$.

We construct an isomorphism of functors between Whit $_{G} \circ F$ and Whit ${ }_{H}$, where $F: \mathrm{D}\left(\operatorname{Bun}_{H}\right) \rightarrow \mathrm{D}\left(\mathrm{Bun}_{G}\right)$ is the theta lifting functor (cf. Theorems 1, 2 and 3$)$.

This result at the level of functions (on $\operatorname{Bun}_{H}(k)$ and $\operatorname{Bun}_{G}(k)$ when $k$ is a finite field) is well known since a long time and the geometrization of the argument is straightforward. We wrote this note for the following reason.

Our proof holds also for $k=\mathbb{C}$ in the setting of $D$-modules. In this case for a reductive group $G$, Beilinson and Drinfeld proposed a conjecture, which (in a form that should be made more precise) says that there exists an equivalence $\alpha_{G}$ between the derived category of D-modules on $\mathrm{Bun}_{G}$ and the derived category of $\theta$-modules on $\operatorname{Loc}_{\check{G}}$. Here $\operatorname{Loc}_{\check{G}}$ is the stack of $\check{G}$-local systems on $X$, and $\check{G}$ is the Langlands dual group to $G$. Moreover, Whit ${ }_{G}$ should be the composition $\mathrm{D}\left(D-\bmod \left(\operatorname{Bun}_{G}\right)\right) \stackrel{\alpha_{G}}{\rightarrow} \mathrm{D}\left(\operatorname{Loc}_{\breve{G}}, \theta\right) \stackrel{\mathrm{R} \Gamma}{\rightarrow} \mathrm{D}(\operatorname{Spec} \mathbb{C})$.

A morphism $\gamma: \check{H} \rightarrow \check{G}$ gives rise to the extension of scalars morphism $\bar{\gamma}: \operatorname{Loc}_{\breve{H}} \rightarrow \operatorname{Loc}_{\check{G}}$. The functor $\bar{\gamma}_{*}: \mathrm{D}\left(\operatorname{Loc}_{\breve{H}}, \theta\right) \rightarrow \mathrm{D}\left(\operatorname{Loc}_{\check{G}}, \theta\right)$ should give rise to the Langlands functoriality functor

$$
\gamma_{L}=\alpha_{G}^{-1} \circ \bar{\gamma}_{*} \circ \alpha_{H}: \mathrm{D}\left(D-\bmod \left(\operatorname{Bun}_{H}\right)\right) \rightarrow \mathrm{D}\left(D-\bmod \left(\operatorname{Bun}_{G}\right)\right)
$$

compatible with the action of Hecke functors.

In the cases $(H, G)=\left(\mathrm{SO}_{2 n}, \mathbb{S p}_{2 n}\right),\left(\mathbb{S p}_{2 n}, \mathrm{SO}_{2 n+2}\right)$ or $\left(\mathbb{G L}_{n}, \mathbb{G L}_{n+1}\right)$ the compatibility of the theta lifting functor $F: \mathrm{D}\left(D-\bmod \left(\operatorname{Bun}_{H}\right)\right) \rightarrow$ $D\left(D-\bmod \left(\operatorname{Bun}_{G}\right)\right)$ with the Hecke functors ([6]) and the compatibility of $F$ with the Whittaker functors (proved in this paper) indicate that $F$ should be the Langlands functoriality functor.

Notation. From now on $k$ denotes an algebraically closed field of characteristic $p>2$, all the stacks we consider are defined over $k$. Let $X$ be a smooth projective curve of genus $g$. Fix a prime $\ell \neq p$ and a non-trivial character $\psi: \mathbb{F}_{p} \rightarrow \overline{\mathbb{Q}}_{\ell}^{*}$, and denote by $\mathscr{L}_{\psi}$ the corresponding Artin-Schreier sheaf on $\mathbb{A}^{1}$. Since $k$ is algebraically closed, we systematically ignore the Tate twists.

For a $k$-stack locally of finite type $S$ write simply $\mathrm{D}(S)$ for the category introduced in ([3], Remark 3.21) and denoted $\mathrm{D}_{c}\left(S, \overline{\mathbb{Q}}_{\ell}\right)$ in loc.cit. It should be thought of as the unbounded derived category of constructible $\overline{\mathbb{Q}}_{\ell}$-sheaves on $S$. For $*=+,-, b$ we have the full triangulated subcategory $\mathrm{D}^{*}(S) \subset \mathrm{D}(S)$ denoted $\mathrm{D}_{c}^{*}\left(S, \overline{\mathbb{Q}}_{\ell}\right)$ in loc.cit. Write $\mathrm{D}^{*}(S) ! \subset \mathrm{D}^{*}(S)$ for the full subcategory of objects which are extensions by zero from some open substack of finite type. Write $\mathrm{D}^{\prec}(S) \subset \mathrm{D}(S)$ for the full subcategory of complexes $K \in D(S)$ such that for any open substack $U \subset S$ of finite type we have $\left.K\right|_{U} \in D^{-}(U)$. 
For any vector space (or bundle) $E$, we define $\operatorname{Sym}^{2}(E)$ and $\Lambda^{2}(E)$ as quotients of $E \otimes E$ (and denote by $x . y$ and $x \wedge y$ the images of $x \otimes y$ ) and we will use in this article the embeddings

$$
\begin{aligned}
& \operatorname{Sym}^{2}(E) \rightarrow E \otimes E \quad \text { and } \Lambda^{2}(E) \rightarrow E \otimes E \\
& x . y \quad \mapsto \frac{x \otimes y+y \otimes x}{2} \quad x \wedge y \mapsto \frac{x \otimes y-y \otimes x}{2}
\end{aligned}
$$

\section{Whittaker functors}

Let $G$ be a reductive group over $k$. We pick a maximal torus and a Borel subgroup $T \subset B \subset G$ and we denote by $\Delta_{G}$ the set of simple roots of $G$. The Whittaker functor

$$
\text { Whit }_{G}: \mathrm{D}^{\prec}\left(\operatorname{Bun}_{G}\right) \rightarrow \mathrm{D}^{-}(\operatorname{Spec} k)
$$

is defined as follows. Write $\Omega$ for the canonical line bundle on $X$. Pick a $T$ torsor $\mathcal{F}_{T}$ on $X$ with a trivial conductor, that is, for each $\check{\alpha} \in \Delta_{G}$ it is equipped with an isomorphism $\delta_{\check{\alpha}}: \mathscr{L}_{\mathscr{F}_{T}}^{\check{\alpha}} \rightarrow \Omega$. Here $\mathscr{L}_{\mathscr{F}_{T}}^{\check{\alpha}}$ is the line bundle obtained from $\mathcal{F}_{T}$ via extension of scalars $T \stackrel{\check{\alpha}}{\rightarrow} \mathbb{G}_{m}$. Let $\operatorname{Bun}_{N}^{\mathcal{F}_{T}}$ be the stack classifying a $B$-torsor $\mathcal{F}_{B}$ together with an isomorphism

$$
\zeta: \mathcal{F}_{B} \times{ }_{B} T \widetilde{\mathcal{F}} \mathcal{F}_{T}
$$

Let $\epsilon: \operatorname{Bun}_{N}^{\mathcal{G}_{T}} \rightarrow \mathbb{A}^{1}$ be the evaluation map (cf. [1], 4.3.1 where it is denoted $\left.e v_{\tilde{\omega}}\right)$. Just recall that for each $\check{\alpha} \in \Delta_{G}$ the class of the extension of $\theta$ by $\Omega$ associated to $\mathcal{F}_{B}, \zeta$ and $\delta_{\check{\alpha}}$ gives $\epsilon_{\check{\alpha}}: \operatorname{Bun}_{N}^{\mathcal{F}_{T}} \rightarrow \mathbb{A}^{1}$ and that $\epsilon=\sum_{\check{\alpha} \in \Delta_{G}} \epsilon_{\check{\alpha}}$. Write $\pi: \operatorname{Bun}_{N}^{\mathcal{F}_{T}} \rightarrow \operatorname{Bun}_{G}$ for the extension of scalars $\left(\mathcal{F}_{B}, \zeta\right) \mapsto \mathcal{F}_{B} \times_{B} G$. Set $P_{\psi}^{0}=\epsilon^{*} \mathscr{L}_{\psi}\left[d_{N}\right]$, where $d_{N}=\operatorname{dim} \operatorname{Bun}_{N}^{\mathscr{F}_{T}}$. Let $d_{G}=\operatorname{dim} \operatorname{Bun}_{G}$. As in ([7], Definition 2) for $\mathcal{F} \in \mathrm{D}^{\prec}\left(\operatorname{Bun}_{G}\right)$ set

$$
\operatorname{Whit}_{G}(\mathcal{F})=\mathrm{R}_{c}\left(\operatorname{Bun}_{N}^{\mathcal{F}_{T}}, P_{\psi}^{0} \otimes \pi^{*}(\mathcal{F})\right)\left[-d_{G}\right]
$$

REMARK 1. - The collection $\left(\mathcal{F}_{T},\left(\delta_{\check{\alpha}}\right)_{\check{\alpha} \in \Delta_{G}}\right)$ as above exists, because $k$ is algebraically closed, and one can take $\mathcal{F}_{T}=(\sqrt{\Omega})^{2 \rho}$ for some square root $\sqrt{\Omega}$ of $\Omega$. One has an exact sequence of abelian group schemes $1 \rightarrow Z \rightarrow T \stackrel{\prod \check{\alpha}}{\longrightarrow}$ $\mathbb{G}_{m}^{\Delta_{G}} \rightarrow 1$, where $Z$ denotes the center of $G$. So, two choices of the collection $\left(\mathcal{F}_{T},\left(\delta_{\check{\alpha}}\right)_{\check{\alpha} \in \Delta_{G}}\right)$ are related by a point of $\operatorname{Bun}_{Z}(k)$ and the associated Whittaker functors are isomorphic up to the automophism of $\mathrm{Bun}_{G}$ given by tensoring with the corresponding $Z$-torsor.

REMARK 2. - When $\mathcal{F}_{T}$ is fixed, the functor Whit ${ }_{G}: \mathrm{D}^{\prec}\left(\mathrm{Bun}_{G}\right) \rightarrow$ $\mathrm{D}^{-}$(Spec $\left.k\right)$ does not depend, up to isomorphism, on the choice of the isomorphisms $\left(\delta_{\check{\alpha}}\right)_{\check{\alpha} \in \Delta_{G}}$. That is, for any $\left(\lambda_{\check{\alpha}}\right)_{\check{\alpha} \in \Delta_{G}} \in\left(k^{*}\right)^{\Delta_{G}}$, the functors associated to $\left(\mathcal{F}_{T},\left(\delta_{\check{\alpha}}\right)_{\check{\alpha} \in \Delta_{G}}\right)$ and $\left(\mathcal{F}_{T},\left(\lambda_{\check{\alpha}} \delta_{\check{\alpha}}\right)_{\check{\alpha} \in \Delta_{G}}\right)$ are isomorphic. Indeed, the two 
diagrams $\operatorname{Bun}_{G} \stackrel{\pi}{\leftarrow} \operatorname{Bun}_{N}^{\mathcal{F}_{T}} \stackrel{\epsilon}{\rightarrow} \mathbb{A}^{1}$ associated to $\left(\delta_{\check{\alpha}}\right)_{\check{\alpha} \in \Delta_{G}}$ and $\left(\lambda_{\check{\alpha}} \delta_{\check{\alpha}}\right)_{\check{\alpha} \in \Delta_{G}}$ are isomorphic for the following reason. Since $k$ is algebraically closed, $T(k) \rightarrow\left(k^{*}\right)^{\Delta_{G}}$ is surjective. We pick any preimage $\gamma \in T(k)$ of $\left(\lambda_{\check{\alpha}}\right)_{\check{\alpha} \in \Delta_{G}}$ and get the automorphism $\left(\mathcal{F}_{B}, \zeta\right) \mapsto\left(\mathcal{F}_{B}, \gamma \zeta\right)$ of $\operatorname{Bun}_{N}^{\mathscr{F}_{T}}$, which together with the idendity of $\operatorname{Bun}_{G}$ and $\mathbb{A}^{1}$ intertwines the two diagrams.

1.1. Whittaker functor for $\mathbb{G L}_{n}$ - For $i, j \in \mathbb{Z}$ with $i \leq j$ we denote by $\mathcal{N}_{i, j}$ the stack classifying the extensions of $\Omega^{i}$ by $\Omega^{i+1} \ldots$ by $\Omega^{j}$, i.e. classifying a vector bundle $E_{j-i+1}$ on $X$ with a complete flag of vector subbundles $0=$ $E_{0} \subset E_{1} \subset \ldots \subset E_{j-i+1}$ together with isomorphisms $E_{k+1} / E_{k} \simeq \Omega^{j-k}$ for $k=0, \ldots, j-i$. Write $\epsilon_{i, j}: \mathcal{N}_{i, j} \rightarrow \mathbb{A}^{1}$ for the map given by the sum of the classes in $\operatorname{Ext}^{1}(\theta, \Omega) \stackrel{\rightarrow}{\rightarrow} \mathbb{A}^{1}$ of the extensions $0 \rightarrow E_{k+1} / E_{k} \rightarrow E_{k+2} / E_{k} \rightarrow$ $E_{k+2} / E_{k+1} \rightarrow 0$ for $k=0, \ldots, j-i-1$.

For $G=\mathbb{G L}_{n}$, we consider the diagram $\operatorname{Bun}_{n} \stackrel{\pi_{0, n-1}}{\leftarrow} \mathcal{N}_{0, n-1} \stackrel{\epsilon_{0, n-1}}{\longrightarrow} \mathbb{A}^{1}$, where $\pi_{0, n-1}: \mathcal{N}_{0, n-1} \rightarrow \operatorname{Bun}_{n}$ is $\left(0=E_{0} \subset \cdots \subset E_{n}\right) \mapsto E_{n}$. This diagram is isomorphic to the diagram $\operatorname{Bun}_{G} \stackrel{\pi}{\leftarrow} \operatorname{Bun}_{N}^{\mathcal{F}_{T}} \stackrel{\epsilon}{\rightarrow} \mathbb{A}^{1}$ associated to the choice of $\mathcal{F}_{T}$ whose image in $\operatorname{Bun}_{n}$ is $\Omega^{n-1} \oplus \Omega^{n-2} \oplus \cdots \oplus \theta$.

Therefore the functor Whit $\mathbb{G L}_{n}: \mathrm{D}^{\prec}\left(\operatorname{Bun}_{n}\right) \rightarrow \mathrm{D}^{-}($Spec $k)$ associated to the above choice of $\mathcal{F}_{T}$ is given by

$\mathrm{Whit}_{\mathbb{G L}}(\mathcal{F})=\mathrm{R} \Gamma_{c}\left(\mathcal{N}_{0, n-1}, \epsilon_{0, n-1}^{*}\left(\mathcal{L}_{\psi}\right) \otimes \pi_{0, n-1}^{*}(\mathcal{F})\right)\left[\operatorname{dim} \mathcal{N}_{0, n-1}-\operatorname{dim} \mathrm{Bun}_{n}\right]$.

REMARK 3. - If $E$ is an irreducible rank $n$ local system on $X$ let Aut $E$ be the corresponding automorphic sheaf on $\operatorname{Bun}_{n}$ (cf. [2]) normalized to be perverse. Then Aut $_{E}$ is equipped with a canonical isomorphism Whit $\mathbb{G L}_{n}\left(\mathrm{Aut}_{E}\right) \widetilde{\rightarrow} \overline{\mathbb{Q}}_{\ell}$. This is our motivation for the above shift normalization in (2).

1.2. Whittaker functor for $\operatorname{Sp}_{2 n}$. - Write $G_{n}$ for the group scheme on $X$ of automorphisms of $\theta^{n} \oplus \Omega^{n}$ preserving the natural symplectic form $\wedge^{2}\left(\theta^{n} \oplus\right.$ $\left.\Omega^{n}\right) \rightarrow \Omega$. The stack Bun $_{G_{n}}$ of $G_{n}$-torsors on $X$ can be seen as the stack classifying vector bundles $M$ over $X$ of rank $2 n$ equipped with a non-degenerate symplectic form $\Lambda^{2} M \rightarrow \Omega$.

The diagram $\operatorname{Bun}_{G_{n}} \stackrel{\pi_{G_{n}}}{\longleftarrow} \mathcal{N}_{G_{n}} \stackrel{\epsilon_{G_{n}}}{\longrightarrow} \mathbb{A}^{1}$ constructed in the next definition is isomorphic to the diagram $\operatorname{Bun}_{G} \stackrel{\pi}{\leftarrow} \operatorname{Bun}_{N}^{\mathscr{F}_{T} \stackrel{\epsilon}{\rightarrow}} \mathbb{A}^{1}$ associated, for $G=G_{n}$, to the choice of $\mathcal{F}_{T}$ whose image in $\operatorname{Bun}_{G_{n}}$ is $L \oplus L^{*} \otimes \Omega$ with $L=\Omega^{n} \oplus \Omega^{n-1} \oplus$ $\cdots \oplus \Omega$ (with the natural symplectic structure for which $L$ and $L^{*} \otimes \Omega$ are lagrangians).

Definition 1. - Let $\mathcal{N}_{G_{n}}$ be the stack classifying $\left(\left(L_{1}, \ldots, L_{n}\right), E\right)$, where $\left(0=L_{0} \subset L_{1} \subset \ldots \subset L_{n}\right) \in \mathcal{N}_{1, n}$, and $E$ is an extension of $\vartheta_{X}$-modules

$$
0 \rightarrow \mathrm{Sym}^{2} L_{n} \rightarrow E \rightarrow \Omega \rightarrow 0
$$


We associate to (3) an extension

$$
0 \rightarrow L_{n} \rightarrow M \rightarrow L_{n}^{*} \otimes \Omega \rightarrow 0
$$

with $M \in \operatorname{Bun}_{G_{n}}$ and $L_{n}$ lagrangian as follows. Equip $L_{n} \oplus L_{n}^{*} \otimes \Omega$ with the symplectic form $\left(l, l^{*}\right),\left(u, u^{*}\right) \mapsto\left\langle l, u^{*}\right\rangle-\left\langle u, l^{*}\right\rangle$ for $l, u \in L, l^{*}, u^{*} \in L^{*}$. Here $\langle.,$.$\rangle is the canonical paring between L_{n}$ and $L_{n}^{*}$. Using (1), we consider (3) as a torsor on $X$ under the sheaf of symmetric morphisms $L_{n}^{*} \otimes \Omega \rightarrow L_{n}$. The latter sheaf acts naturally on $L_{n} \oplus L_{n}^{*} \otimes \Omega$ preserving the symplectic form. Then $M$ is the twisting of $L_{n} \oplus L_{n}^{*} \otimes \Omega$ by the above torsor. This defines a morphism $\pi_{G_{n}}: \mathcal{N}_{G_{n}} \rightarrow \operatorname{Bun}_{G_{n}}$.

Note that the extension of $\Omega$ by $L_{n} \otimes L_{n}$ obtained from (4) is the pushforward of (3) by the embedding $\operatorname{Sym}^{2} L_{n} \rightarrow L_{n} \otimes L_{n}$ we have fixed in (1).

Let $\epsilon_{G_{n}}: \mathcal{N}_{G_{n}} \rightarrow \mathbb{A}^{1}$ denote the sum of $\epsilon_{1, n}\left(L_{1}, \ldots, L_{n}\right)$ with the class in $\operatorname{Ext}(\Theta, \Omega)=\mathbb{A}^{1}$ of the push-forward of (3) by $\operatorname{Sym}^{2} L_{n} \rightarrow \operatorname{Sym}^{2}\left(L_{n} / L_{n-1}\right)=$ $\Omega^{2}$.

The functor Whit $_{G_{n}}: \mathrm{D}^{\prec}\left(\operatorname{Bun}_{G_{n}}\right) \rightarrow \mathrm{D}^{-}($Spec $k)$ associated to the above choice of $\mathcal{F}_{T}$ is given by

$$
\operatorname{Whit}_{G_{n}}(\mathcal{F})=\mathrm{R} \Gamma_{c}\left(\mathcal{N}_{G_{n}}, \epsilon_{G_{n}}^{*}\left(\mathscr{L}_{\psi}\right) \otimes \pi_{G_{n}}^{*}(\mathcal{F})\right)\left[d_{N\left(G_{n}\right)}-d_{G_{n}}\right]
$$

with $d_{N\left(G_{n}\right)}=\operatorname{dim} \mathcal{N}_{G_{n}}$ and $d_{G_{n}}=\operatorname{dim} \operatorname{Bun}_{G_{n}}$.

1.3. Whittaker functor for $\mathrm{SO}_{2 n}$ (first form). - Let $H_{n}=\mathrm{SO}_{2 n}$. The stack Bun $_{H_{n}}$ of $H_{n}$-torsors can be seen as the stack classifying vector bundles $V$ over $X$ equipped with a non-degenerate symmetric form $\operatorname{Sym}^{2} V \rightarrow \theta$ and a compatible trivialization $\operatorname{det} V \stackrel{\rightarrow}{\rightarrow}$.

The diagram $\operatorname{Bun}_{H_{n}} \stackrel{\pi_{H_{n}}}{\longleftarrow} \mathcal{N}_{H_{n}} \stackrel{\epsilon_{H_{n}}}{\longrightarrow} \mathbb{A}^{1}$ constructed in the next definition is isomorphic to the diagram $\operatorname{Bun}_{G} \stackrel{\pi}{\leftarrow} \operatorname{Bun}_{N}^{\mathcal{F}_{T}} \stackrel{\epsilon}{\rightarrow} \mathbb{A}^{1}$ associated, for $G=H_{n}$, to the choice of $\mathcal{F}_{T}$ whose image in $\operatorname{Bun}_{H_{n}}$ is $U \oplus U^{*}$ with $U=\Omega^{n-1} \oplus \Omega^{n-2} \oplus \cdots \oplus$ $\vartheta$ (with the natural symmetric structure for which $U$ and $U^{*}$ are isotropic).

Definition 2. - Let $\mathcal{N}_{H_{n}}$ be the stack classifying $\left(\left(U_{1}, \ldots, U_{n}\right), E\right)$, where $\left(U_{1}, \ldots, U_{n}\right) \in \mathcal{N}_{0, n-1}$ (i.e. we have a filtration $0=U_{0} \subset U_{1} \subset \ldots \subset U_{n}$ with $U_{i} / U_{i-1} \simeq \Omega^{n-i}$ for $\left.i=1, \ldots, n\right)$, and $E$ is an extension of $\theta_{X}$-modules

$$
0 \rightarrow \Lambda^{2} U_{n} \rightarrow E \rightarrow \theta \rightarrow 0
$$

We associate to (5) an extension

$$
0 \rightarrow U_{n} \rightarrow V \rightarrow U_{n}^{*} \rightarrow 0
$$

with $V \in \operatorname{Bun}_{H_{n}}$ and $U_{n}$ isotropic as follows. Equip $U_{n} \oplus U_{n}^{*}$ with the symmetric form given by $\left(u, u^{*}\right),\left(v, v^{*}\right) \mapsto\left\langle u, v^{*}\right\rangle+\left\langle v, u^{*}\right\rangle$ with $u, v \in U_{n}, u^{*}, v^{*} \in U_{n}^{*}$. Using (1), we consider (5) as a torsor under the sheaf of antisymmetric morphisms $U_{n}^{*} \rightarrow U_{n}$ of $\theta_{X}$-modules. This sheaf acts naturally on $U_{n} \oplus U_{n}^{*}$ preserving the 
symmetric form and the trivialization of $\operatorname{det}\left(U_{n} \oplus U_{n}^{*}\right)$. Then (6) is the twisting of $U_{n} \oplus U_{n}^{*}$ by the above torsor. This defines a morphism $\pi_{H_{n}}: \mathcal{N}_{H_{n}} \rightarrow \operatorname{Bun}_{H_{n}}$.

Note that the extension of $\theta_{X}$ by $U_{n} \otimes U_{n}$ obtained from (6) is the pushforward of (5) by the embedding $\Lambda^{2} U_{n} \rightarrow U_{n} \otimes U_{n}$ fixed in (1).

For $\lambda \in k^{*}$ let $\epsilon_{H_{n}, \lambda}: \mathcal{N}_{H_{n}} \rightarrow \mathbb{A}^{1}$ be the sum of $\epsilon_{0, n-1}\left(U_{1}, \ldots, U_{n}\right)$ with $\lambda u$, where $u \in \operatorname{Ext}(\theta, \Omega)=\mathbb{A}^{1}$ is the class of the push-forward of (5) by $\Lambda^{2} U_{n} \rightarrow \Lambda^{2}\left(U_{n} / U_{n-2}\right)=\Omega$. Set $\epsilon_{H_{n}}=\epsilon_{H_{n}, 1}$.

The functor Whit $H_{n}: \mathrm{D}^{\prec}\left(\operatorname{Bun}_{H_{n}}\right) \rightarrow \mathrm{D}^{-}(\operatorname{Spec} k)$ associated to the above choice of $\mathcal{F}_{T}$ sends $\mathcal{F} \in D^{\prec}\left(\operatorname{Bun}_{H_{n}}\right)$ to

$$
\operatorname{Whit}_{H_{n}}(\mathcal{F})=\mathrm{R} \Gamma_{c}\left(\mathcal{N}_{H_{n}}, \epsilon_{H_{n}}^{*}\left(\mathscr{L}_{\psi}\right) \otimes \pi_{H_{n}}^{*}(M)\right)\left[d_{N\left(H_{n}\right)}-d_{H_{n}}\right]
$$

with $d_{N\left(H_{n}\right)}=\operatorname{dim} \mathcal{N}_{H_{n}}$ and $d_{H_{n}}=\operatorname{dim} \operatorname{Bun}_{H_{n}}$. By Remark 2, if we replace in (7) $\epsilon_{H_{n}}$ by $\epsilon_{H_{n}, \lambda}$ then the functor Whit $H_{n}$ gets replaced by an isomorphic one.

\subsection{Whittaker functor for $\mathrm{SO}_{2 n}$ (second form)}

Definition 3. - Let $\widetilde{\mathcal{N}}_{H_{n}}$ be the stack classifying $\left(V_{1} \subset \cdots \subset V_{n} \subset V\right)$, where $V \in \operatorname{Bun}_{H_{n}}, V_{n} \subset V$ is a subbundle, $\left(V_{1}, \ldots, V_{n}\right) \in \mathcal{N}_{0, n-1}$ (i.e. we have a filtration $0=V_{0} \subset V_{1} \subset \ldots \subset V_{n}$ with $V_{i} / V_{i-1} \simeq \Omega^{n-i}$ for $\left.i=1, \ldots, n\right)$, and the composition

$$
\mathrm{Sym}^{2} V_{n} \rightarrow \mathrm{Sym}^{2} V \rightarrow \theta
$$

coincides with $\operatorname{Sym}^{2} V_{n} \rightarrow \operatorname{Sym}^{2}\left(V_{n} / V_{n-1}\right)=\theta$ (in particular $V_{n-1}$ is isotropic).

The morphism $\widetilde{\pi}_{H_{n}}: \widetilde{\mathcal{N}}_{H_{n}} \rightarrow \operatorname{Bun}_{H_{n}}$ sends $\left(\left(V_{1}, \ldots, V_{n}\right), V\right)$ to $V$. The morphism $\widetilde{\epsilon}_{H_{n}}: \widetilde{\mathcal{N}}_{H_{n}} \rightarrow \mathbb{A}^{1}$ is given by $\widetilde{\epsilon}_{H_{n}}\left(\left(V_{1}, \ldots, V_{n}\right), V\right)=\epsilon_{0, n-1}\left(V_{1}, \ldots, V_{n}\right)$.

Define a morphism $\kappa: \mathcal{N}_{H_{n}} \rightarrow \widetilde{\mathcal{N}}_{H_{n}}$ as follows. Let $\left.\left(U_{1}, \ldots, U_{n}\right), E\right) \in \mathcal{N}_{H_{n}}$ and let $V$ be as in Definition 2. For $i=1, \ldots, n-1$ define $V_{i}$ as the image of $U_{i}$ in $V$ and $V_{2 n-i}$ as the orthogonal of $V_{i}$ in $V$. Then we have a filtration

$$
0=V_{0} \subset V_{1} \subset \ldots \subset V_{n-1} \subset V_{n+1} \subset \ldots \subset V_{2 n-1} \subset V_{2 n}=V .
$$

Recall that we have an identification $U_{n} / U_{n-1} \simeq \theta$. The exact sequence $0 \rightarrow$ $U_{n} / U_{n-1} \rightarrow V_{n+1} / V_{n-1} \rightarrow V_{n+1} / U_{n} \rightarrow 0$ admits a unique splitting $s$ such that the image of $\theta=V_{n+1} / U_{n} \stackrel{s}{\rightarrow} V_{n+1} / V_{n-1}$ is isotropic. Thus, $V_{n+1} / V_{n-1}$ is canonically identified with $\theta \oplus \theta$ in such a way that the symmetric bilinear form $\operatorname{Sym}^{2}(\theta \oplus \theta) \rightarrow \theta$ becomes

$$
(1,0) .(1,0) \mapsto 0,(1,0) .(0,1) \mapsto 1,(0,1) .(0,1) \mapsto 0
$$

Under this identification $\theta=U_{n} / U_{n-1} \rightarrow V_{n+1} / V_{n-1}=\theta \oplus \Theta$ sends 1 to $(1,0)$.

Define $V_{n}$, equipped with $\theta \simeq V_{n} / V_{n-1}$ by the property that $\theta \simeq$ $V_{n} / V_{n-1} \hookrightarrow V_{n+1} / V_{n-1}$ sends 1 to $\left(1, \frac{1}{2}\right) \in \Theta \oplus \Theta$. The following is easy to check. 
LEMma 1. - The map $\kappa: \mathcal{N}_{H_{n}} \rightarrow \widetilde{\mathcal{N}}_{H_{n}}$ is an isomorphism. There exists $\lambda \in k^{*}$ such that $\widetilde{\epsilon}_{H_{n}} \circ \kappa=\epsilon_{H_{n}, \lambda}$ and $\widetilde{\pi}_{H_{n}} \circ \kappa=\pi_{H_{n}}$.

By Remark 2, if we replace in (7) $\epsilon_{H_{n}}, \pi_{H_{n}}$ by $\tilde{\epsilon}_{H_{n}}, \tilde{\pi}_{H_{n}}$ then the functor Whit $_{H_{n}}$ gets replaced by an isomorphic one.

\section{Main statements}

Write $\operatorname{Bun}_{n}$ for the stack of rank $n$ vector bundles on $X$. Let $\operatorname{Bun}_{P_{n}}$ be the stack classifying $L \in \operatorname{Bun}_{n}$ and an exact sequence $0 \rightarrow \operatorname{Sym}^{2} L \rightarrow ? \rightarrow \Omega \rightarrow 0$. Remind the complex $S_{P, \psi}$ on Bun $_{P_{n}}$ introduced in ([4], 5.2). Let $\mathcal{V}$ be the stack over $\operatorname{Bun}_{n}$ whose fibre over $L$ is $\operatorname{Hom}(L, \Omega)$. For $\chi_{n}=V \times_{\operatorname{Bun}_{n}} \operatorname{Bun}_{P_{n}}$ let $p: \chi_{n} \rightarrow \operatorname{Bun}_{P_{n}}$ be the projection. Write $q: \chi_{n} \rightarrow \mathbb{A}^{1}$ for the map sending $s \in \operatorname{Hom}(L, \Omega)$ to the pairing of $s \otimes s \in \operatorname{Hom}\left(\operatorname{Sym}^{2} L, \Omega^{2}\right)$ with the exact sequence $0 \rightarrow \operatorname{Sym}^{2} L \rightarrow ? \rightarrow \Omega \rightarrow 0$. Let $d_{\chi_{n}}$ be the "corrected" dimension of $\chi_{n}$, i.e. the locally constant function $\operatorname{dim} \operatorname{Bun}_{P_{n}}-\chi(L)$. Set

$$
S_{P, \psi}=p_{!} q^{*} \mathscr{L}_{\psi}\left[d_{\chi_{n}}\right]
$$

Let $\mathscr{C}$ be the line bundle on $\operatorname{Bun}_{G_{n}}$ whose fibre at $M$ is $\operatorname{det} \mathrm{R} \Gamma(X, M)$. Write $\widetilde{\operatorname{Bun}}_{G_{n}}$ for the gerb of square roots of $\mathscr{G}$ and Aut for the theta-sheaf on $\widetilde{\operatorname{Bun}}_{G_{n}}$ ([4], Definition 1). The projection $\nu_{n}: \operatorname{Bun}_{P_{n}} \rightarrow \operatorname{Bun}_{G_{n}}$ lifts naturally to a map $\tilde{\nu}_{n}: \operatorname{Bun}_{P_{n}} \rightarrow \widehat{\operatorname{Bun}}_{G_{n}}$. In what follows, we pick an isomorphism(1)

$$
\left.S_{P, \psi} \widetilde{\rightarrow} \tilde{\nu}_{n}^{*} \operatorname{Aut}\left[\operatorname{dim} \cdot \operatorname{rel}\left(\tilde{\nu}_{n}\right)\right)\right]
$$

provided by ([5], Proposition 1). Here $\operatorname{dim} \cdot \operatorname{rel}\left(\tilde{\nu}_{n}\right)$ is the relative dimension of $\tilde{\nu}_{n}$. The isomorphisms we construct below may depend on this choice.

2.1. From $\mathbb{S p}_{2 n}$ to $\mathrm{SO}_{2 n+2}$. - Let $F: \mathrm{D}^{-}\left(\operatorname{Bun}_{G_{n}}\right) ! \rightarrow \mathrm{D}^{\prec}\left(\operatorname{Bun}_{H_{n+1}}\right)$ be the theta lifting functor introduced in ([6], Definition 2).

THeorem 1. - The functors Whit $H_{n+1} \circ F$ and Whit $_{G_{n}}$ from $\mathrm{D}^{-}\left(\mathrm{Bun}_{G_{n}}\right)$ ! to $\mathrm{D}^{-}(\operatorname{Spec} k)$ are isomorphic.

Let $\chi$ be the stack classifying $\left(M,\left(U_{1}, \ldots, U_{n+1}\right), E, s\right)$ with $M \in \operatorname{Bun}_{G_{n}}$, $\left(U_{1}, \ldots, U_{n+1}\right) \in \mathcal{N}_{0, n}$ (i.e. $U_{k+1} / U_{k}=\Omega^{n-k}$ for $\left.k=0, \ldots, n\right), E$ an extension $0 \rightarrow \Lambda^{2} U_{n+1} \rightarrow E \rightarrow \Theta \rightarrow 0$, and $s: U_{n+1} \rightarrow M$ a morphism of $\Theta_{X}$-modules.

Let $\alpha_{\chi}: \chi \rightarrow \operatorname{Bun}_{G_{n}}$ be the morphism $\left(M,\left(U_{1}, \ldots, U_{n+1}\right), E, s\right) \mapsto M$. Let $\beta_{\chi}: \chi \rightarrow \mathbb{A}^{1}$ be defined as follows. For $\left(M,\left(U_{1}, \ldots, U_{n+1}\right), E, s\right) \in \chi$,

$$
\beta_{\chi}\left(M,\left(U_{1}, \ldots, U_{n+1}\right), E, s\right)=\epsilon_{0, n}\left(U_{1}, \ldots, U_{n+1}\right)+\gamma(E)-\left\langle E, \Lambda^{2} s\right\rangle
$$

(1) Once $\sqrt{-1} \in k$ is chosen, this isomorphism is well defined up to a sign. 
where $\gamma(E)$ is the pairing between the class of $E$ in $\operatorname{Ext}\left(\theta, \Lambda^{2} U_{n+1}\right)$ and the morphism $\Lambda^{2} U_{n+1} \rightarrow \Lambda^{2}\left(U_{n+1} / U_{n-1}\right)=\Omega$ and $\left\langle E, \Lambda^{2} s\right\rangle$ is the pairing between the class of $E$ in $\operatorname{Ext}\left(\theta, \Lambda^{2} U_{n+1}\right)$ and $\Lambda^{2} s: \Lambda^{2} U_{n+1} \rightarrow \Lambda^{2} M$ followed by $\Lambda^{2} M \rightarrow \Omega$.

Let $a_{n}=n(n+1)(1-g)\left(n-\frac{1}{2}\right)$, this is the dimension of the stack classifying extension $0 \rightarrow \wedge^{2} U_{n+1} \rightarrow ? \rightarrow \Theta \rightarrow 0$ of $\theta_{X}$-modules for any fixed $\left(U_{1}, \ldots, U_{n+1}\right) \in \mathcal{N}_{0, n}$.

Let $d_{\alpha_{\chi}}$ denote the "corrected" relative dimension of $\alpha_{\chi}$, that is, $d_{\alpha_{\chi}}=$ $a_{n}+\operatorname{dim} \mathcal{N}_{0, n}+\chi\left(U_{n+1}^{*} \otimes M\right)$ for any $k$-points $M \in \operatorname{Bun}_{G_{n}}$ and $\left(U_{1}, \ldots, U_{n+1}\right) \in$ $\mathcal{N}_{0, n}$. One checks that (8) yields for $\mathcal{F} \in \mathrm{D}^{-}\left(\operatorname{Bun}_{G_{n}}\right)$ ! an isomorphism in $\mathrm{D}^{-}(\operatorname{Spec} k)$

$$
\text { Whit }_{H_{n+1}} \circ F(\mathcal{F}) \stackrel{\rightarrow}{\rightarrow} \Gamma_{c}\left(\chi, \alpha_{\chi}^{*}(\mathcal{F}) \otimes \beta_{\chi}^{*}\left(\mathscr{L}_{\psi}\right)\left[d_{\alpha_{\chi}}\right]\right)
$$

We will show later that Theorem 1 is reduced to the following proposition.

Proposition 1. - There is an isomorphism $\alpha_{\chi !}\left(\beta_{\chi}^{*}\left(\mathscr{L}_{\psi}\right)\left[2 a_{n}\right]\right) \widetilde{\rightarrow} \pi_{G_{n} !} \epsilon_{G_{n}}^{*}\left(\mathscr{L}_{\psi}\right)$ in $\mathrm{D}^{-}\left(\operatorname{Bun}_{G_{n}}\right) ! \cdot$

The proposition is a consequence of the following lemmas. Let $y$ be the stack classifying $\left(M,\left(U_{1}, \ldots, U_{n+1}\right), s\right)$ with $M \in \operatorname{Bun}_{G_{n}},\left(U_{1}, \ldots, U_{n+1}\right) \in \mathcal{N}_{0, n}$ (i.e. $U_{k+1} / U_{k}=\Omega^{n-k}$ for $\left.k=0, \ldots, n\right)$, and $s: U_{n+1} \rightarrow M$ a morphism such that the composition $\Lambda^{2} U_{n+1} \stackrel{\Lambda^{2} s}{\rightarrow} \Lambda^{2} M \rightarrow \Omega$ coincides with $\Lambda^{2} U_{n+1} \rightarrow$ $\Lambda^{2}\left(U_{n+1} / U_{n-1}\right)=\Omega$.

Let $\alpha y: y \rightarrow \operatorname{Bun}_{G_{n}}$ be the morphism $\left(M,\left(U_{1}, \ldots, U_{n+1}\right), s\right) \mapsto M$. Let $\beta_{y}$ : $y \rightarrow \mathbb{A}^{1}$ be the map sending $\left(M,\left(U_{1}, \ldots, U_{n+1}\right), s\right) \in \mathcal{Y}$ to $\epsilon_{0, n}\left(U_{1}, \ldots, U_{n+1}\right)$.

LEMMA 2. - There is an isomorphism $\alpha_{\chi, !} \beta_{\chi}^{*}\left(\mathscr{L}_{\psi}\right)=\alpha_{y_{!} !} \beta_{y}^{*}\left(\mathscr{L}_{\psi}\right)\left[-2 a_{n}\right]$ in $\mathrm{D}^{-}\left(\operatorname{Bun}_{G_{n}}\right) !$.

For $i \in\{1, \ldots, n+1\}$ let $\mathscr{Y}_{i}$ denote the open subset of $\mathscr{Y}$ given by the condition that the image of $U_{i}$ by $s$ is a subbundle of $M$. One has open

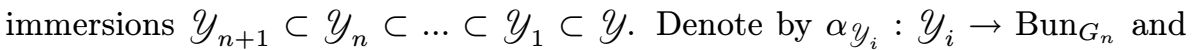
$\beta_{y_{i}}: y_{i} \rightarrow \mathbb{A}^{1}$ the restrictions of $\alpha y$ and $\beta_{y}$ to $y_{i}$.

LEMMA 3. - The natural maps $\alpha_{y_{n+1}, !} \beta_{y_{n+1}^{*}}\left(\mathscr{L}_{\psi}\right) \rightarrow \alpha_{y_{n}, !} \beta_{y_{n}}^{*}\left(\mathscr{L}_{\psi}\right) \rightarrow \ldots \rightarrow$ $\alpha_{y_{1}, !} \beta_{y_{1}}^{*}\left(\mathscr{L}_{\psi}\right) \rightarrow \alpha_{y_{1} !} \beta_{y}^{*}\left(\mathscr{L}_{\psi}\right)$ are isomorphisms in $\mathrm{D}^{-}\left(\operatorname{Bun}_{G_{n}}\right) !$

Proof. - First, one has $\mathscr{Y}_{n+1}=\mathscr{Y}_{n-1}$ thanks to the condition that the composition $\Lambda^{2} U_{n+1} \stackrel{\Lambda^{2} s}{\rightarrow} \Lambda^{2} M \rightarrow \Omega$ coincides with $\Lambda^{2} U_{n+1} \rightarrow \Lambda^{2}\left(U_{n+1} / U_{n-1}\right)=\Omega$.

Write $\mathscr{Y}_{0}=\mathcal{Y}$. Let $i \in\{1, \ldots, n-1\}$. We are going to prove that the natural map

$$
\alpha_{y_{i}, !} \beta_{y_{i}}^{*}\left(\mathscr{L}_{\psi}\right) \rightarrow \alpha_{y_{i-1}, !} \beta_{y_{i-1}}^{*}\left(\mathscr{L}_{\psi}\right)
$$


is an isomorphism. Set $Z_{i}=Y_{i-1} \backslash Y_{i}$, let $\alpha_{Z_{i}}$ and $\beta_{Z_{i}}$ be the restrictions of $\alpha_{y_{i-1}}$ and $\beta_{y_{i-1}}$ to $Z_{i}$. We must prove that $\alpha_{Z_{i}, !} \beta_{Z_{i}}^{*}\left(\mathcal{L}_{\psi}\right)=0$.

Let $\mathcal{T}_{i}$ be stack classifying $\left(M,\left(U_{1}, U_{2}, \ldots, U_{i}\right), s_{i}\right)$ with $M \in \operatorname{Bun}_{G_{n}}$, $\left(U_{1}, U_{2}, \ldots, U_{i}\right) \in \mathcal{N}_{n-i+1, n}, s_{i}: U_{i} \rightarrow M$ such that the restriction of $s_{i}$ to $U_{i-1}$ is injective and its image is a subbundle of $M$, but the image of $s_{i}$ is not a subbundle of $M$ of the same rank as $U_{i}$. The map $\alpha_{Z_{i}}$ decomposes naturally as $Z_{i} \stackrel{\gamma_{Z_{i}}}{\rightarrow} \mathcal{T}_{i} \stackrel{\alpha_{\mathcal{G}_{i}}}{\rightarrow} \operatorname{Bun}_{G_{n}}$. It suffices to show that the $*$-fibre of $\gamma_{Z_{i}, !} \beta_{Z_{i}}^{*}\left(\mathscr{L}_{\psi}\right)$ at any closed point $\left(M,\left(U_{1}, U_{2}, \ldots, U_{i}\right), s_{i}\right) \in \mathcal{J}_{i}$ vanishes.

The fiber $\mathcal{Q}$ of $\gamma_{Z_{i}}$ over this point is the stack classifying $\left(\left(U_{1}, \ldots, U_{n+1}\right), s\right)$, where $\left(U_{1}, \ldots, U_{n+1}\right) \in \mathcal{N}_{0, n}$ extends $\left(U_{1}, U_{2}, \ldots, U_{i}\right), s: U_{n+1} \rightarrow M$ extends $s_{i}$, and the composition $\Lambda^{2} U_{n+1} \stackrel{\Lambda^{2} s}{\rightarrow} \Lambda^{2} M \rightarrow \Omega$ coincides with $\Lambda^{2} U_{n+1} \rightarrow$ $\Lambda^{2}\left(U_{n+1} / U_{n-1}\right)=\Omega$.

Let $F$ denote the smallest subbundle of $M$ containing $s\left(U_{i}\right)$, its rank is $i$ or $i-1$. Let $\mathcal{R}$ be stack classifying $\left(\left(W_{1}, \ldots, W_{n+1-i}\right), t\right)$ with $\left(W_{1}, \ldots, W_{n+1-i}\right) \in$ $\mathcal{N}_{0, n-i}$ and $t \in \operatorname{Hom}\left(W_{n+1-i}, M / F\right)$. There is a morphism $\rho: \mathcal{Q} \rightarrow \mathcal{R}$ which sends $\left(\left(U_{1}, \ldots, U_{n+1}\right), s\right)$ to $\left(\left(U_{i+1} / U_{i}, \ldots, U_{n+1} / U_{i}\right), \bar{s}\right)$ where $\bar{s}: U_{n+1} / U_{i} \rightarrow$ $M / F$ is the reduction of $s$. Let $\beta_{Q}: Q \rightarrow \mathbb{A}^{1}$ be the restriction of $\beta_{Z_{i}}$ to $Q$. It suffices to show that $\rho_{!} \beta_{Q}^{*}\left(\mathscr{L}_{\psi}\right)=0$.

Pick $\left(\left(W_{1}, \ldots, W_{n+1-i}\right), t\right) \in \mathscr{R}$, let $\&$ be the fiber of $\rho$ over

$$
\left(\left(W_{1}, \ldots, W_{n+1-i}\right), t\right) \text {. }
$$

Write $\beta_{\phi}$ for the restriction of $\beta_{Q}$ to $\phi$. We will show that $R \Gamma_{c}\left(\phi, \beta_{\phi}^{*}\left(\mathcal{L}_{\psi}\right)\right)=0$.

If $F$ is of rank $i-1$ then $\&$ identifies with the stack classifying extensions $0 \rightarrow U_{i} / U_{i-1} \rightarrow ? \rightarrow U_{n+1} / U_{i} \rightarrow 0$ of $\theta_{X}$-modules. Since $\beta_{\S}$ is a nontrivial character, we are done in this case.

If $F$ is of rank $i$ then $\&$ is a scheme with a free transitive action of $\operatorname{Hom}\left(U_{n+1} / U_{i}, F / s\left(U_{i}\right)\right)$. Under the action of $\operatorname{Hom}\left(U_{n+1} / U_{i}, F / s\left(U_{i}\right)\right), \beta_{\phi}$ changes by some character

$$
\operatorname{Hom}\left(U_{n+1} / U_{i}, F / s\left(U_{i}\right)\right) \rightarrow \operatorname{Hom}\left(U_{i+1} / U_{i}, F / s\left(U_{i}\right)\right) \stackrel{\delta}{\rightarrow} \mathbb{A}^{1} .
$$

If $D=\operatorname{div}\left(F / s\left(U_{i}\right)\right)$ then $F / s\left(U_{i}\right) \widetilde{\rightarrow} \Omega^{n-i+1}(D) / \Omega^{n-i+1}$ naturally, and $\delta$ : $\mathrm{H}^{0}(X, \Omega(D) / \Omega) \rightarrow \mathrm{H}^{1}(X, \Omega)$ is the map induced by the short exact sequence $0 \rightarrow \Omega \rightarrow \Omega(D) \rightarrow \Omega(D) / \Omega \rightarrow 0$, i.e. it is the sum of the residues. Since $D>0$, $\delta$ is nontrivial, and we are done.

LEMma 4. - There is an isomorphism $\mu: \mathscr{Y}_{n+1} \rightarrow \mathcal{N}_{G_{n}}$ such that $\pi_{G_{n}} \circ \mu=$ $\alpha y_{n+1}$ and $\epsilon_{G_{n}} \circ \mu=\beta_{y_{n+1}}$.

It remains to show that Proposition 1 implies Theorem 1 . By the base change theorem we have

$$
\mathrm{Whit}_{G_{n}}(\mathcal{F}) \stackrel{\rightarrow}{\rightarrow} R \Gamma_{c}\left(\mathcal{N}_{G_{n}}, \epsilon_{G_{n}}^{*}\left(\mathscr{L}_{\psi}\right) \otimes \pi_{G_{n}}^{*}(\mathcal{F})\right)\left[d_{N\left(G_{n}\right)}-d_{G_{n}}\right]
$$




$$
\stackrel{\sim}{\rightarrow} R \Gamma_{c}\left(\operatorname{Bun}_{G_{n}}, \pi_{G_{n}, !} \epsilon_{G_{n}}^{*}\left(\mathcal{L}_{\psi}\right) \otimes \mathcal{F}\right)\left[d_{N\left(G_{n}\right)}-d_{G_{n}}\right]
$$

and

$$
R \Gamma_{c}\left(\chi, \alpha_{\chi}^{*}(\mathcal{F}) \otimes \beta_{\chi}\left(\mathscr{L}_{\psi}\right)\left[d_{\alpha_{\chi}}\right]\right) \stackrel{\sim}{\rightarrow} R \Gamma_{c}\left(\operatorname{Bun}_{G_{n}}, \alpha_{\chi, !}\left(\beta_{\chi}^{*}\left(\mathscr{L}_{\psi}\right) \otimes \mathcal{F}\left[d_{\alpha_{\chi}}\right]\right)\right) .
$$

It remains to prove $d_{\alpha_{\chi}}-2 a_{n}=d_{N\left(G_{n}\right)}-d_{G_{n}}$. This follows from $d_{G_{n}}=$ $-(1-g) n(2 n+1), d_{N\left(G_{n}\right)}-\operatorname{dim} \mathcal{N}_{0, n}=(1-g)\left(-n^{2}+n(n+1)\left(n-\frac{1}{2}\right)\right)$, and $\chi\left(U_{n+1}^{*} \otimes M\right)=(1-g) 2 n^{2}(n+1)$ where $\left(U_{1}, \ldots, U_{n+1}\right)$ and $M$ are closed points in $\mathcal{N}_{0, n}$ and $\operatorname{Bun}_{G_{n}}$.

2.2. From $\mathrm{SO}_{2 n}$ to $\mathrm{Sp}_{2 n}$. - Let $F: \mathrm{D}^{-}\left(\operatorname{Bun}_{H_{n}}\right) ! \rightarrow \mathrm{D}^{\prec}\left(\operatorname{Bun}_{G_{n}}\right)$ be the Theta functor introduced in ([6], Definition 2).

TheOREM 2. - The functors Whit $_{G_{n}} \circ F$ and Whit $_{H_{n}}$ from $\mathrm{D}^{-}\left(\mathrm{Bun}_{H_{n}}\right)$ ! to $\mathrm{D}^{-}(\mathrm{Spec} k)$ are isomorphic.

We use the same letters as in the last paragraph (with a different meaning), as the proof is very similar.

Let $\chi$ be the stack classifying $\left(V,\left(L_{1}, \ldots, L_{n}\right), E, s\right)$ with $V \in \operatorname{Bun}_{H_{n}}$, $\left(L_{1}, \ldots, L_{n}\right) \in \mathcal{N}_{1, n}$ (i.e. $L_{k+1} / L_{k}=\Omega^{n-k}$ for $\left.k=0, \ldots, n-1\right)$, an extension $0 \rightarrow \operatorname{Sym}^{2} L_{n} \rightarrow E \rightarrow \Omega \rightarrow 0$ of $\theta_{X}$-modules, and a section $s: L_{n} \rightarrow V \otimes \Omega$.

Let $\alpha_{\chi}: \chi \rightarrow \operatorname{Bun}_{H_{n}}$ be the morphism $\left(V,\left(L_{1}, \ldots, L_{n}\right), E, s\right) \mapsto V$. Let $\beta_{\chi}: \chi \rightarrow \mathbb{A}^{1}$ be the map sending $\left(V,\left(L_{1}, \ldots, L_{n}\right), E, s\right) \in \chi$ to

$$
\epsilon_{1, n}\left(L_{1}, \ldots, L_{n}\right)+\gamma(E)-\left\langle E, \operatorname{Sym}^{2} s\right\rangle,
$$

where $\gamma(E)$ is the pairing between the class of $E$ in $\operatorname{Ext}^{1}\left(\Omega, \operatorname{Sym}^{2} L_{n}\right)$ and the $\operatorname{map} \operatorname{Sym}^{2} L_{n} \rightarrow \operatorname{Sym}^{2}\left(L_{n} / L_{n-1}\right)=\Omega^{2} ;\left\langle E, \operatorname{Sym}^{2} s\right\rangle$ is the pairing between the class of $E$ in $\operatorname{Ext}^{1}\left(\Omega, \operatorname{Sym}^{2} L_{n}\right)$ and $\operatorname{Sym}^{2} s: \operatorname{Sym}^{2} L_{n} \rightarrow \operatorname{Sym}^{2} V \otimes \Omega^{2}$ followed by $\operatorname{Sym}^{2} V \rightarrow \vartheta$.

Let $b_{n}=-\chi\left(\Omega^{-1} \otimes \operatorname{Sym}^{2} L_{n}\right)$ for any $k$-point $\left(L_{1}, \ldots, L_{n}\right) \in \mathcal{N}_{1, n}$. Write $d_{\alpha \chi}$ for the "corrected" relative dimension of $\alpha_{\chi}$, that is,

$$
d_{\alpha_{\chi}}=\operatorname{dim} \mathcal{N}_{1, n}+b_{n}+\chi\left(L_{n}^{*} \otimes V \otimes \Omega\right)
$$

for any $k$-points $\left(L_{1}, \ldots, L_{n}\right) \in \mathcal{N}_{1, n}$ and $V \in \operatorname{Bun}_{H_{n}}$. One checks that (8) yields for $\mathcal{F} \in \mathrm{D}^{-}\left(\operatorname{Bun}_{H_{n}}\right)$ ! an isomorphism in $\mathrm{D}^{-}(\operatorname{Spec} k)$

$$
\text { Whit }_{G_{n}} \circ F(\mathcal{F})=\mathrm{R} \Gamma_{c}\left(\chi, \alpha_{\chi}^{*}(\mathcal{F}) \otimes \beta_{\chi}^{*}\left(\mathscr{L}_{\psi}\right)\right)\left[d_{\alpha_{\chi}}\right]
$$

We will derive Theorem 2 from the following proposition.

Proposition 2. - There is an isomorphism $\alpha_{\chi, !} \beta_{\chi}^{*}\left(\mathscr{L}_{\psi}\right)\left[2 b_{n}\right] \simeq \widetilde{\pi}_{H_{n}, !} \tilde{\epsilon}_{H_{n}}^{*}\left(\mathscr{L}_{\psi}\right)$ in $\mathrm{D}^{-}\left(\operatorname{Bun}_{H_{n}}\right)$ ! 
Proposition 2 is reduced to the following lemmas. Let $y$ be the stack classifying $\left(V,\left(L_{1}, \ldots, L_{n}\right), s\right)$ with $V \in \mathrm{Bun}_{\mathrm{SO}_{2 n}},\left(L_{1}, \ldots, L_{n}\right) \in \mathcal{N}_{1, n}$ (i.e., $L_{k+1} / L_{k}=\Omega^{n-k}$ for $\left.k=0, \ldots, n-1\right)$ and $s: L_{n} \rightarrow V \otimes \Omega$ a morphism such that the composition $\mathrm{Sym}^{2} L_{n} \stackrel{\mathrm{Sym}^{2} s}{\longrightarrow}\left(\mathrm{Sym}^{2} V\right) \otimes \Omega^{2} \rightarrow \Omega^{2}$ coincides with

$$
\operatorname{Sym}^{2} L_{n} \rightarrow \operatorname{Sym}^{2}\left(L_{n} / L_{n-1}\right)=\Omega^{2}
$$

Let $\alpha_{y}: y \rightarrow \operatorname{Bun}_{H_{n}}$ be the map $\left(V,\left(L_{1}, \ldots, L_{n}\right), s\right) \mapsto V$. Let $\beta_{y}: y \rightarrow \mathbb{A}^{1}$ be the map sending $\left(V,\left(L_{1}, \ldots, L_{n}\right), s\right) \in \mathcal{Y}$ to $\epsilon_{1, n}\left(L_{1}, \ldots, L_{n}\right)$.

LEMMA 5. - There is an isomorphism $\alpha_{\chi, !} \beta_{\chi}^{*}\left(\mathscr{L}_{\psi}\right) \widetilde{\rightarrow} \alpha_{Y}, \beta_{Y}^{*}\left(\mathscr{L}_{\psi}\right)\left[-2 b_{n}\right]$ in $\mathrm{D}^{-}\left(\operatorname{Bun}_{H_{n}}\right)$ !

For $i \in\{1, \ldots, n\}$ let $\mathscr{Y}_{i} \subset \mathcal{Y}$ be the open substack given by the condition that $s\left(L_{i}\right) \subset V \otimes \Omega$ is a subbundle of rank $i$. We have inclusions $\mathscr{Y}_{n} \subset \mathscr{Y}_{n-1} \subset \ldots \subset$ $y_{1} \subset y_{\text {. Denote by }} \alpha_{y_{i}}: y_{i} \rightarrow \operatorname{Bun}_{H_{n}}$ and $\beta_{y_{i}}: y_{i} \rightarrow \mathbb{A}^{1}$ the restrictions of $\alpha y$ and $\beta_{y}$ to $y_{i}$.

As in Lemma 3, one proves

LEMMA 6. - The natural maps $\alpha_{y_{n}, !} \beta_{y_{n}}^{*}\left(\mathscr{L}_{\psi}\right) \rightarrow \alpha_{y_{n-1}, !} \beta_{y_{n-1}}^{*}\left(\mathscr{L}_{\psi}\right) \rightarrow \ldots \rightarrow$

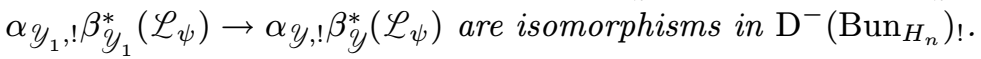

LEMMA 7. - There is an isomorphism $\mu: \mathscr{Y}_{n} \rightarrow \widetilde{\mathcal{N}}_{\mathrm{SO}_{2 n}}$ such that $\widetilde{\pi}_{\mathrm{SO}_{2 n}} \circ \mu=$ $\alpha y_{n}$ and $\widetilde{\epsilon}_{\mathrm{SO}_{2 n}} \circ \mu=\beta_{y_{n}}$.

Theorem 2 follows from Proposition 2 because $d_{\alpha_{\chi}}-2 b_{n}=d_{N\left(H_{n}\right)}-d_{H_{n}}$. Let us just indicate that $d_{N\left(H_{n}\right)}-\operatorname{dim} \mathcal{N}_{1, n}=(1-g) n(n-1)\left(n-\frac{3}{2}\right), \chi\left(L_{n}^{*} \otimes V \otimes \Omega\right)=$ $(1-g) 2 n^{3}, b_{n}=(1-g) n(n+1)\left(n-\frac{1}{2}\right)$ and $d_{H_{n}}=-(1-g) n(2 n-1)$, where $\left(L_{1}, \ldots, L_{n}\right)$ and $V$ are closed points in $\mathcal{N}_{1, n}$ and $\operatorname{Bun}_{H_{n}}$.

2.3. From $\mathbb{G L}_{n}$ to $\mathbb{G L}_{n+1}$. - Let $F: \mathrm{D}^{-}\left(\mathrm{Bun}_{n}\right) ! \rightarrow \mathrm{D}^{\prec}\left(\mathrm{Bun}_{n+1}\right)$ be the composition of the direct image by $\mathrm{Bun}_{n} \rightarrow \mathrm{Bun}_{n}, L \mapsto L^{*}$ and the theta functor $F_{n, n+1}: \mathrm{D}^{-}\left(\operatorname{Bun}_{n}\right) ! \rightarrow \mathrm{D}^{\prec}\left(\operatorname{Bun}_{n+1}\right)$ introduced in ([6], Definition 3). It is a consequence of Theorem 5 in [6] that $F$ is compatible with Hecke functors according to the morphism of dual groups $\mathbb{G L}_{n} \rightarrow \mathbb{G L}_{n+1}, A \mapsto\left(\begin{array}{ll}A & 0 \\ 0 & 1\end{array}\right)$.

Let us recall the definition of $F$. Denote $W$ be the classifying stack of $(L, U, s)$ with $L \in \mathrm{Bun}_{n}, U \in \mathrm{Bun}_{n+1}$ and $s: L \rightarrow U$ a morphism. We have $\left(h_{n}, h_{n+1}\right): \mathcal{W} \rightarrow \operatorname{Bun}_{n} \times \operatorname{Bun}_{n+1},(L, U, s) \mapsto(L, U)$. Then for $\mathcal{F} \in$ $\mathrm{D}^{-}\left(\operatorname{Bun}_{n}\right) !$

$$
F(\mathcal{F})=h_{n+1, !}\left(\left(h_{n}^{*} \mathcal{F}\right)\left[\operatorname{dim} \operatorname{Bun}_{n+1}+\chi\left(L^{*} \otimes U\right)\right]\right),
$$

where $\chi\left(L^{*} \otimes U\right)$ is considered as a locally constant function on $\operatorname{Bun}_{n} \times \operatorname{Bun}_{n+1}$. 
THEOREM 3. - The functors Whit $\mathbb{G L}_{n+1} \circ F$ and Whit $_{\mathbb{G L}_{n}}$ from $\mathrm{D}^{-}\left(\operatorname{Bun}_{n}\right)$ ! to $\mathrm{D}^{-}(\operatorname{Spec} k)$ are isomorphic.

Let $\chi$ be the stack classifying $L \in \operatorname{Bun}_{n},\left(U_{1}, \ldots, U_{n+1}\right) \in \mathcal{N}_{0, n}$, and $s$ : $L \rightarrow U_{n+1}$ a morphism. We have $\alpha_{\chi}: \chi \rightarrow \operatorname{Bun}_{n}$ and $\beta_{\chi}: \chi \rightarrow \mathbb{A}^{1}$ which send $\left(L,\left(U_{1}, \ldots U_{n+1}\right), s\right)$ to $L$ and $\epsilon_{0, n}\left(U_{1}, \ldots U_{n+1}\right)$.

We have

Whit $_{\mathbb{G L}_{n+1}} \circ F(\mathcal{F})=\mathrm{R}_{c}\left(\operatorname{Bun}_{n}, \mathcal{F} \otimes \alpha_{\chi, !} \beta_{\chi}^{*}\left(\mathscr{L}_{\psi}\right)\left[\operatorname{dim} \mathcal{N}_{0, n}+\chi\left(L^{*} \otimes U_{n+1}\right)\right]\right)$ and Whit $_{\mathbb{G L}_{n}}(\mathcal{F})=\mathrm{R}_{c}\left(\operatorname{Bun}_{n}, \mathcal{F} \otimes\left(\pi_{0, n-1}\right) ! \epsilon_{0, n-1}^{*}\left(\mathcal{L}_{\psi}\right)\left[\operatorname{dim} \mathcal{N}_{0, n-1}-\operatorname{dim} \operatorname{Bun}_{n}\right]\right)$.

For $i \in\{0, \ldots, n\}$ denote by $\chi_{i}$ the open substack of $\chi$ classifying $\left(L,\left(U_{1}, \ldots U_{n+1}\right), s\right)$ such that the composition $L \stackrel{s}{\rightarrow} U_{n+1} \rightarrow U_{n+1} / U_{n+1-i}$ is surjective. We have $\chi=\chi_{0} \supset \chi_{1} \supset \ldots \supset \chi_{n}$ and we have an isomorphism $\mathcal{N}_{0, n-1} \rightarrow \chi_{n}$ which sends $\left(E_{1}, \ldots, E_{n}\right)$ to $\left(E_{n},\left(\Omega^{n}, \Omega^{n} \oplus E_{1}, \ldots, \Omega^{n} \oplus\right.\right.$ $\left.E_{n}\right),(0$, Id $\left.)\right)$ with $(0$, Id $): E_{n} \rightarrow \Omega^{n} \oplus E_{n}$ the obvious inclusion.

It is easy to compute that for $L=E_{n}$ with $\left(E_{1}, \ldots, E_{n}\right) \in \mathcal{N}_{0, n-1}$ and $\left(U_{1}, \ldots, U_{n+1}\right) \in \mathcal{N}_{0, n}$ we have $\operatorname{dim} \mathcal{N}_{0, n}+\chi\left(L^{*} \otimes U_{n+1}\right)=\operatorname{dim} \mathcal{N}_{0, n-1}-$ $\operatorname{dim} \operatorname{Bun}_{n}$.

Therefore we are reduced to the following lemma. We denote by $\alpha_{\chi_{i}}: \chi_{i} \rightarrow$ $\operatorname{Bun}_{n}$ and $\beta_{\chi_{i}}: \chi_{i} \rightarrow \mathbb{A}^{1}$ the restrictions of $\alpha_{\chi}$ and $\beta_{\chi}$ to $\chi_{i}$.

LEMMA 8. - The natural maps $\alpha_{\chi_{n}, !} \beta_{\chi_{n}}^{*}\left(\mathscr{L}_{\psi}\right) \rightarrow \alpha_{\chi_{n-1}, !} \beta_{\chi_{n-1}}^{*}\left(\mathscr{L}_{\psi}\right) \rightarrow \ldots \rightarrow$ $\alpha_{\chi_{1}, !} \beta_{\chi_{1}}^{*}\left(\mathcal{L}_{\psi}\right) \rightarrow \alpha_{\chi, !} \beta_{\chi}^{*}\left(\mathscr{L}_{\psi}\right)$ are isomorphisms in $\mathrm{D}^{-}\left(\operatorname{Bun}_{n}\right) ! \cdot$

Proof. - We recall that $\chi=\chi_{0}$. Let $i \in\{1, \ldots, n\}$. We are going to prove that the natural map

$$
\alpha_{\chi_{i}, !} \beta_{\chi_{i}}^{*}\left(\mathscr{L}_{\psi}\right) \rightarrow \alpha_{\chi_{i-1}, !} \beta_{\chi_{i-1}}^{*}\left(\mathscr{L}_{\psi}\right)
$$

is an isomorphism. Set $Z_{i}=\chi_{i-1} \backslash \chi_{i}$, let $\alpha_{Z_{i}}$ and $\beta_{Z_{i}}$ be the restrictions of $\alpha_{\chi_{i-1}}$ and $\beta_{\chi_{i-1}}$ to $Z_{i}$. We must prove that $\alpha_{Z_{i}, !} \beta_{Z_{i}}^{*}\left(\mathcal{L}_{\psi}\right)=0$.

Let $\mathcal{J}_{i}$ be stack classifying $\left(L,\left(V_{1}, V_{2}, \ldots, V_{i}\right), t\right)$ with $L \in \operatorname{Bun}_{n}$, $\left(V_{1}, V_{2}, \ldots, V_{i}\right) \in \mathcal{N}_{0, i-1}, t: L \rightarrow V_{i}$ such that the composition $L \stackrel{t}{\rightarrow}$ $V_{i} \rightarrow V_{i} / V_{1}$ is surjective but $t$ is not surjective. The map $\alpha_{Z_{i}}$ decomposes naturally as $Z_{i} \stackrel{\gamma_{Z_{i}}}{\rightarrow} \mathcal{T}_{i} \stackrel{\alpha_{\mathcal{G}_{i}}}{\rightarrow} \operatorname{Bun}_{n}$ where $\gamma_{Z_{i}}\left(L,\left(U_{1}, \ldots U_{n+1}\right), s\right)=$ $\left(L,\left(U_{n+2-i} / U_{n+1-i}, \ldots, U_{n+1} / U_{n+1-i}\right), \bar{s}\right)$ and $\alpha_{\mathcal{J}_{i}}\left(L,\left(U_{1}, \ldots U_{n+1}\right), s\right)=L$. It suffices to show that the $*$-fibre of $\gamma_{Z_{i}, !} \beta_{Z_{i}}^{*}\left(\mathcal{L}_{\psi}\right)$ at any closed point $\left(L,\left(V_{1}, V_{2}, \ldots, V_{i}\right), t\right) \in \mathcal{J}_{i}$ vanishes.

Let us choose a closed point $\left(L,\left(V_{1}, V_{2}, \ldots, V_{i}\right), t\right) \in \mathcal{J}_{i}$ and define $L^{\prime \prime}=\operatorname{Ker} t$ and $L^{\prime}$ the kernel of the composition $L \stackrel{t}{\rightarrow} V_{i} \rightarrow V_{i} / V_{1}$. Then $L^{\prime}$ is a subbundle of $L$ of rank $n+1-i$ and $L^{\prime \prime}$ is a subbundle of $L$ of $\operatorname{rank} n+1-i$ or $n-i$. 
The fiber $Q$ of $\gamma_{Z_{i}}$ over this closed point is the stack classifying $\left(\left(U_{1}, \ldots, U_{n+1}\right), s\right)$, with an isomorphism between $U_{n+1} / U_{n+1-i}$ and $V_{i}$ sending $U_{j+n+1-i} / U_{n+1-i}$ to $V_{j}$ for any $j \in\{0, \ldots, i\}$ and $s: L \rightarrow U_{n+1}$ such that the composition $L \stackrel{s}{\rightarrow} U_{n+1} \rightarrow U_{n+1} / U_{n+1-i} \simeq V_{i}$ is $t$. Let $\mathscr{R}$ be stack classifying $\left(\left(U_{1}, \ldots, U_{n+1-i}\right), s_{i}\right)$ with $\left(U_{1}, \ldots, U_{n+1-i}\right) \in \mathcal{N}_{i, n}$ and $s_{i} \in \operatorname{Hom}\left(L^{\prime \prime}, U_{n+1-i}\right)$. There is a morphism $\rho: \mathcal{Q} \rightarrow \mathcal{R}$ which sends $\left(\left(U_{1}, \ldots, U_{n+1}\right), s\right)$ to $\left(\left(U_{1}, \ldots, U_{n+1-i}\right), s_{i}\right)$ where $s_{i}$ is the restriction of $s$ to $L^{\prime \prime}$. Let $\beta_{Q}: Q \rightarrow \mathbb{A}^{1}$ be the restriction of $\beta_{Z_{i}}$ to $Q$. It suffices to show that $\rho_{!} \beta_{Q}^{*}\left(\mathscr{L}_{\psi}\right)=0$.

Pick $\left(\left(U_{1}, \ldots, U_{n+1-i}\right), s_{i}\right) \in \mathscr{R}$, let $\&$ be the fiber of $\rho$ over $\left(\left(U_{1}, \ldots, U_{n+1-i}\right), s_{i}\right)$. Write $\beta_{\&}$ for the restriction of $\beta_{Q}$ to $\&$. We will show that $R \Gamma_{c}\left(\varnothing, \beta_{\delta}^{*}\left(\mathcal{L}_{\psi}\right)\right)=0$.

If $L^{\prime}=L^{\prime \prime}$ we have an exact sequence $0 \rightarrow L / L^{\prime \prime} \rightarrow U_{n+1} / U_{n+1-i} \rightarrow$ $U_{n+2-i} / U_{n+1-i} \rightarrow 0$, and $\&$ identifies with the stack classifying extensions $0 \rightarrow$ $U_{n+1-i} \rightarrow ? \rightarrow U_{n+2-i} / U_{n+1-i} \rightarrow 0$ of $\theta_{X}$-modules. Since $\beta_{\&}$ is a nontrivial character, we are done in this case.

If $L^{\prime} / L^{\prime \prime}$ is a line bundle then $\&$ is a scheme with a free transitive action of the $H^{0}$ of the cone of the morphism of complexes of $k$-vector spaces

$$
\operatorname{RHom}\left(U_{n+1} / U_{n+1-i}, U_{n+1-i}\right) \rightarrow \operatorname{RHom}\left(L / L^{\prime \prime}, U_{n+1-i}\right)
$$

which is also the cone of the morphism of complexes

$$
\operatorname{RHom}\left(U_{n+2-i} / U_{n+1-i}, U_{n+1-i}\right) \rightarrow \operatorname{RHom}\left(L^{\prime} / L^{\prime \prime}, U_{n+1-i}\right)
$$

and whose cohomology is concentrated in degree 0 . The last morphism of complexes comes from the non zero morphism $L^{\prime} / L^{\prime \prime} \rightarrow U_{n+2-i} / U_{n+1-i}=$ $\Omega^{i-1}$ which identifies $L^{\prime} / L^{\prime \prime}$ to $\Omega^{i-1}(-D)$ for some effective non zero divisor $D$. Therefore the $H^{0}$ of this cone is equal to

$$
H^{0}\left(X, U_{n+1-i} \otimes \Omega^{1-i}(D) / U_{n+1-i} \otimes \Omega^{1-i}\right)
$$

and $\beta_{\phi}^{*}\left(\mathscr{L}_{\psi}\right)$ transforms under this action through the character

$$
\begin{gathered}
H^{0}\left(X, U_{n+1-i} \otimes \Omega^{1-i}(D) / U_{n+1-i} \otimes \Omega^{1-i}\right) \rightarrow \\
H^{0}\left(X,\left(U_{n+1-i} / U_{n-i}\right) \otimes \Omega^{1-i}(D) /\left(U_{n+1-i} / U_{n-i}\right) \otimes \Omega^{1-i}\right)=H^{0}(X, \Omega(D) / \Omega) \stackrel{\sigma}{\rightarrow} \mathbb{A}^{1}
\end{gathered}
$$

where $\sigma$ is the sum of the residues. Since $D$ is non zero, $\sigma$ is a non zero character and we are done.

\section{BIBLIOGRAPHY}

[1] E. Frenkel, D. Gaitsgory \& K. Vilonen - "Whittaker patterns in the geometry of moduli spaces of bundles on curves", Ann. of Math. 153 (2001), p. 699-748. 
[2] _ , "On the geometric Langlands conjecture", J. Amer. Math. Soc. 15 (2002), p. 367-417.

[3] Y. Laszlo \& M. Olsson - "The six operations for sheaves on Artin stacks. II. Adic coefficients", Publ. Math. Inst. Hautes Études Sci. 107 (2008), p. 169-210.

[4] S. Lysenko - "Moduli of metaplectic bundles on curves and thetasheaves", Ann. Sci. École Norm. Sup. 39 (2006), p. 415-466.

[5] _ , "Geometric Waldspurger periods", Compos. Math. 144 (2008), p. $377-438$.

[6] _ "Geometric theta-lifting for the dual pair $\mathbb{S O}_{2 m}, \mathbb{S}_{2 n}$ ", Ann. Sci. École Norm. Sup. 44 (2011), p. 427-493.

[7] — "On automorphic sheaves on Bun $_{G}$ ", preprint arXiv:math/0211067. 